\title{
Simulations of oxidation of metal nanoparticles with a grain boundary inside
}

\author{
Vladimir P. Zhdanov ${ }^{1,2}$ (D)
}

Received: 20 February 2020 / Accepted: 1 July 2020 / Published online: 12 July 2020

(C) The Author(s) 2020

\begin{abstract}
The generic 2D lattice Monte Carlo simulations presented herein are focused on the spatio-temporal kinetics of oxidation of metal nanoparticles composed of two grains separated by a single grain boundary. The oxidation is assumed to occur via inward diffusion of interstitial oxygen ions in the oxide. The results of simulations illustrate that the regimes of oxidation can range from one where the presence of grains is negligible and the oxide shell is formed at the periphery of a whole nanoparticle to one where each grain is oxidized almost independently.
\end{abstract}

Keywords Nanoparticles · Oxidation kinetics · Diffusion · Grains · Grain boundaries

\section{Introduction}

Oxidation of macroscopic metal samples occurs via formation of a surface oxide layer and subsequent growth of an oxide film mediated by diffusion of metal and oxygen ions through this film. Due to its high practical importance, this process has long attracted attention, and the corresponding experimental and theoretical studies can be tracked for many decades (reviewed in [1, 2]; for the generic models, see Refs. [3-11]). Nowadays, this area is still open for research (see, e.g., recent experiments [12-16] and models [17-25]) due to its complexity related to (i) generation of the electric field between the metal-oxide and oxide-gas interfaces, (ii) stress (or lattice strain) arising due to the lattice expansion during oxide formation, (iii) numerous defects in the oxide structure and their evolution, e.g., via grain growth, and (iv) defects, e.g., grain boundaries and dislocations in the metal phase.

Electronic supplementary material The online version of this article (https://doi.org/10.1007/s1114 4-020-01818-4) contains supplementary material, which is available to authorized users.

Vladimir P. Zhdanov

zhdanov@chalmers.se

1 Department of Physics, Chalmers University of Technology, Göteborg, Sweden

2 Boreskov Institute of Catalysis, Russian Academy of Sciences, Novosibirsk, Russia 
With rapid development of nanoscience during the past decade, many studies have become to be focused on oxidation of metal nanoparticles (NPs; see, e.g., recent experiments [26-34] and models [35-43]). Such NPs can often be easily fully converted into the oxide state, and accordingly the understanding of the mechanisms of their oxidation and its specifics compared to the macroscopic samples is interesting not only from the perspective of basic science but also crucial for their numerous applications in various fields ranging from electronics to medicine. The extent of complexity in this area is comparable with or even larger than that observed in the case of macroscopic samples. In particular, the above-mentioned factors complicating the interpretation of oxidation of macroscopic samples operate in the case of NPs as well, and their description is more challenging due to nonplanar geometry. In addition, one can e.g. notice that the oxidation of metal NPs is often accompanied by the formation of holes inside (Kirkendall effect) [44, 45]. In macroscopic samples, the role of this effect is usually considered to be minor. The understanding of the mechanistic details of oxidation of metal NPs is now limited because accurate monitoring of oxidation on the nm scale is still challenging (see, e.g., already mentioned recent studies [26-34]).

The experimental studies of oxidation of metals were/are accompanied by the development of the kinetic models allowing one to understand and interpret what happens or may happen in the reality. Nowadays, such models are numerous, and as a rule each one is focused on one of the scenarios of oxidation. Historically, the theory was long aimed at the formation of an oxide film on the surface of macroscopic metal samples [1-11]. During the past decade, some of the corresponding models have been generalized in order to describe oxidation of metal NPs as briefly outlined below.

The simplest model of oxidation of metals is based on the assumption that the process is limited by conventional Fickian diffusion of metal and/or oxygen ions via the oxide film. The one-dimensional (1D) version of this model proposed by Tammann [3] and Pilling and Bedworth [4] describes oxidation of macroscopic metal samples and predicts the parabolic law for the film thickness, $l \propto t^{1 / 2}$. For spherically shaped particles, the corresponding analytical treatment was given by Valensi [6] and Carter [8]. In fact, this was the first model applicable (with some reservations) to describing oxidation of metal NPs (see, e.g., its use in [30]).

The rate of metal oxidation can depend on the electric field generated in the oxide film because this field induces the drift of metal and oxygen ions through this film. The first 1D model describing this effect was proposed by Wagner [5] (for one of the first and recent extensions of this model, see Refs. [9, 19], respectively). The model implies that the field is weak in the sense that it does not influence the ion diffusion coefficients and mobilities, and the oxidation kinetics was predicted to be parabolic. The alternative 1D model implying that the field is strong was proposed by Cabrera and Mott [7]. Recently, the latter model was generalized to describe metal NPs and nanowires (Refs. [38-40] and [43]).

The volume of oxide formed during metal oxidation is appreciably larger than the volume of metal which is converted to oxide. This mismatch of the volumes is accommodated by oxide reconfiguration and can be accompanied by the creation of stress in an oxide and metal, formation and growth of grains in an oxide, and 
formation of cracks there. In general, diffusion along line and surface defects including dislocations, grain boundaries, and cracks is well known to be often facilitated [46-48], and the presence and/or generation of such defects can influence the oxidation kinetics. The models describing various related aspects of metal oxidation are of different categories. The simplest phenomenological models are focused on grain growth in an oxide and the role of diffusion via the corresponding grain boundaries. The first model of this category was proposed for macroscopic metal samples by Fehlner and Mott [10] (its generalization to NPs is given in [41]). Two examples of recent models are in Ref. [24, 25]. The simplest model describing the formation of cracks is presented in Ref. [11]. The effect of stress on oxidation of macroscopic metal samples was analyzed by employing various phenomenological (e.g. phasefield) models [20-23]. Various approximations ware used to describe the Kirkendall effect accompanied oxidation of metal NPs (Refs. [35-37] and [42, 43]).

As already noticed in the beginning, the oxide formation can be influenced by the presence of grains in a metal. The corresponding experimental studies related to macroscopic samples are, however, not numerous, the results obtained are diverse, and their interpretation is not straightforward. For example, the oxidation of polycrystalline $\mathrm{Ni}$ (the process is presumably controlled by diffusion of $\mathrm{Ni}$ ions) was found to be appreciably faster than that of $\mathrm{Ni}(100)$ [49]. This might indicate that in the former case the ion transport occurred mostly along grain boundaries in $\mathrm{Ni}$. The small spot and imaging studies did not, however, show localized growth at these boundaries. In the case of oxidation of thin Ni films, the kinetics were observed to be insensitive to the size of grains in $\mathrm{Ni}$ [50]. On the other hand, Maack and Nilius [16] have recently found (by using the UV/Vis transmission spectroscopy) that in contrast to classical oxidation theories the oxidation of polycrystalline $\mathrm{Cu}$ films shows an accelerated behavior, being explained by the formation of a corrugated metal-oxide interface due to preferred oxidation along grain boundaries in $\mathrm{Cu}$.

In metal NPs, the rate of relaxation of grain boundaries or dislocations rapidly increases with decreasing particle size $[51,52]$. In relatively large particles of size $\sim 100 \mathrm{~nm}$, grains can be fairly stable, and the pathway of oxidation along grain boundaries in a metal can be efficient. Herein, I present Monte Carlo (MC) simulations clarifying the likely features of this channel of oxide formation in metal NPs. The model employed is focused on the interplay of oxygen diffusion through the oxide shell, oxygen diffusion via the grain boundary in the metal core, and the oxide formation at the metal-oxide interface. In this framework, the regimes of oxidation may range from one where the presence of grains is negligible and the oxide shell is formed at the periphery of a NP to one where each grain is oxidized almost independently. The simulations presented illustrate the transition between these limits and identify the conditions of their realization.

\section{Model}

As already noticed in "Introduction", the oxidation of metal NPs depends on numerous factors, and attempts to include simultaneously many of them into a model make its analysis hardly possible. As many other models of metal oxidation in general 
and oxidation of metal NPs in particular, the model employed herein is focused on one of the factors, i.e., on the role of grain boundaries in a metal. This channel of oxidation is expected to be especially important when the process is controlled by inward diffusion of interstitial oxygen ions (IOIs) via the oxide, because oxygen can in this case move along grain boundaries in a metal and form a complex oxide front (by analogy with that observed in [16]). The MC technique is especially suitable for simulations of propagation of such fronts provided a model is sufficiently simplified in order to perform MC simulations.

In the model under consideration, the self-consisting simplifications are related to the structure and kinetic steps. Concerning the kinetic steps, the model implies that the oxidation is fully controlled by inward diffusion of IOIs. The outward diffusion of metal ions is neglected. The hollow formation in the metal (Kirkendall effect), related usually to the latter diffusion, is accordingly neglected as well. The other structural simplifications are based on the lattice approximation. In particular, a NP is represented by a 2D $L_{x} \times L_{y}$ square lattice. Qualitatively, this lattice can be viewed as a cross-section of a nanocrystallite. Each lattice site can be in one of two states representing metal or oxide, respectively. IOIs are allowed to be in the sites representing oxide. Each such site can contain only one IOI. Initially, the whole particle is assumed to be either in a single-crystal state (no grain boundaries) or viewed as a composite containing two equivalent grains with a grain boundary between them. In the latter case, the grain boundary is represented by two vertical row of sites located in the center (the corresponding horizontal coordinates are $i=L_{x} / 2$ and $L_{x} / 2+1$ ).

The formation of the first oxide layer at the external boundary is considered to be rapid, and initially (at $t=0$ ) the external-boundary sites are prescribed to be in the oxide state. IOIs located at these sites are assumed to be at thermodynamic equilibrium with the gas-phase oxygen. This equilibrium is mimicked by using the grand canonical distribution for IOAs. Practically, this means that during a MC trial involving a boundary site it is prescribed either to have an IOI with probability $p_{\mathrm{b}} \ll 1$ or to be vacant with probability $1-p_{\mathrm{b}}$. This probability represents the average occupation of the external-boundary sites by IOAs. In reality, $p_{\mathrm{b}}$ is proportional to $P_{\mathrm{O}_{2}}^{1 / 2}$, where $P_{\mathrm{O}_{2}}$ is the oxygen pressure. Physically, the assumption that oxygen on the gas-oxide interface is at equilibrium is reasonable because the rates of the rate processes occurring at the interface are usually much faster than that of the whole oxidation process. In various models of oxidation of metals, this assumption is widely used since the first theoretical studies mentioned in "Introduction".

The oxidation of the grain-boundary sites is considered to be rapid as well, and at $t=0$ these sites are also prescribed to be in the oxide state. Except the external- and grain-boundary sites, all other sites represent metal at $t=0$.

Diffusion of IOIs and conversion of the sites from the metal state to the oxide state are realized via attempts of IOA jumps to nearest-neighbour (nn) sites. The effect of electric field and lattice strain on these processes is neglected. This is acceptable provided a NP is not too small and the oxide shell is able to relax during its formation. In reality, these factors may of course influence the oxidation kinetics. Their inclusion into the analysis can appreciably complicate the simulations but is not expected to change the main conclusions drawn below. 
In the model under consideration, IOIs can diffuse at the regular oxide sites and the external- and grain-boundary oxide sites. In reality, the rates of diffusion on such sites are different. The difference of the rates of diffusion on the regular and external-boundary oxide sites is, however, not expected to be important, because in reality the surface and subsurface layers usually do not limit the metal oxidation. In the model employed, as already noticed, the IOIs located at the external-boundary oxide sites are assumed to be at thermodynamic equilibrium with the gas-phase oxygen. This implies that the jumps of IOIs along the external boundary are rapid. The rate constants of the IOI jumps between these sites and the nn regular oxide sites are considered to be equal.

For IOI diffusion along the grain-boundary oxide sites, I analyzed two scenarios. The first one implies that this diffusion is rapid, and accordingly IOIs located at these sites are at thermodynamic equilibrium with the gas-phase oxygen and this equilibrium is described by using the grand canonical distribution (exactly as in the case of the external-boundary sites). In the second (opposite) and more realistic scenario, the rate constants of the IOI diffusion jumps at the grain-boundary oxide sites are considered to be the same as on the regular oxide sites. In both scenarios, the rate constants of the IOA jumps between the grain-boundary sites and the nn regular oxide sites are assumed to be equal.

\section{Algorithm of simulations}

With the specification above, the algorithm of the MC simulations is as follows:

(i) A site is chosen at random.

(ii) If the site selected is vacant, a trial ends.

(iii) If the site is occupied, the IOI located in this site tries to diffuse or to form oxide. In particular, a nn site is randomly selected, and if the latter site is occupied by an IOI, the trial ends. If the nn site is in the oxide state and vacant, the IOI jumps to it with unit probability. If the nn site is in the metal state, the oxidation event, including removal of the chosen IOI with simultaneous conversion of the nn site from the metal state to the oxide state is realized with probability $p_{\mathrm{ox}}$.

(iv) After each MC trial, the time is incremented by $\Delta t=|\ln (\rho)| /\left(L_{x} \times L_{y}\right)$, where $0<\rho \leq 1$ is a random number.

On average, $\Delta t=1$ corresponds to $L_{x} \times L_{y}$ MC trials. In the simulations presented, $\Delta t=1$ is identified, as usual, with one MC step (MCS). To convert $t$ into real time, it should be divided by the properly defined jump rate constant. For my goals, the time units are not important, and I measure time in MCS.

The simulations were performed on a lattice with $L_{x}=140$ and $L_{y}=100$. The external-boundary oxide sites were described by using $p_{\mathrm{b}}=0.1$. The rate constant of the conversion of the sites from the metal state to the oxide state, $p_{\text {ox }}$, was varied in a wide range in order to illustrate various regimes of the oxidation kinetics. 


\section{Results of simulations}

Typical results of simulations are presented in Figs. 1, 2, 3, 4, 5, and 6. In particular, Fig. 1 shows five representative kinetics of oxidation, whereas Figs. 2, 3, 4, 5 and 6 exhibit and the corresponding lattice snapshots at the moment when

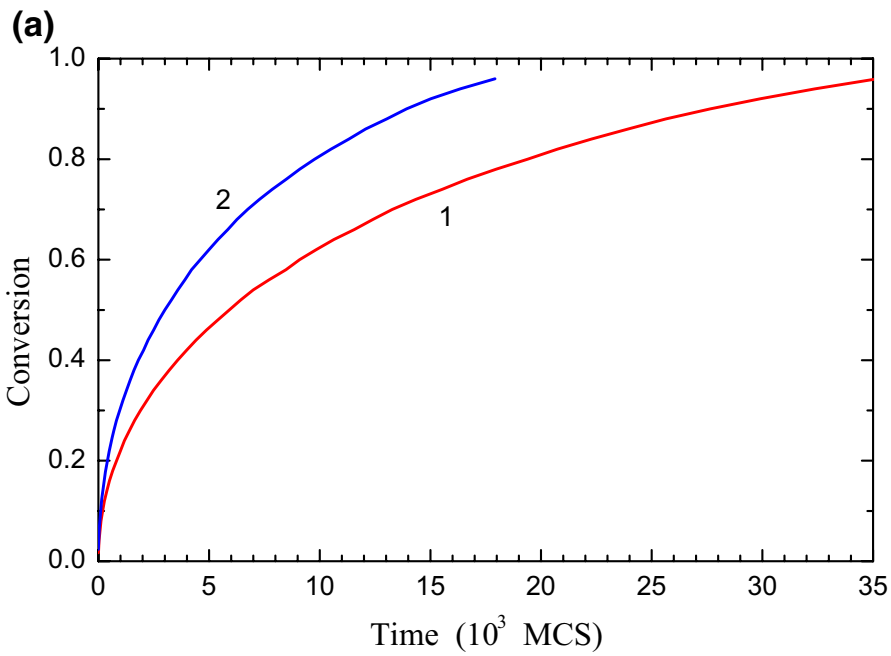

(b)

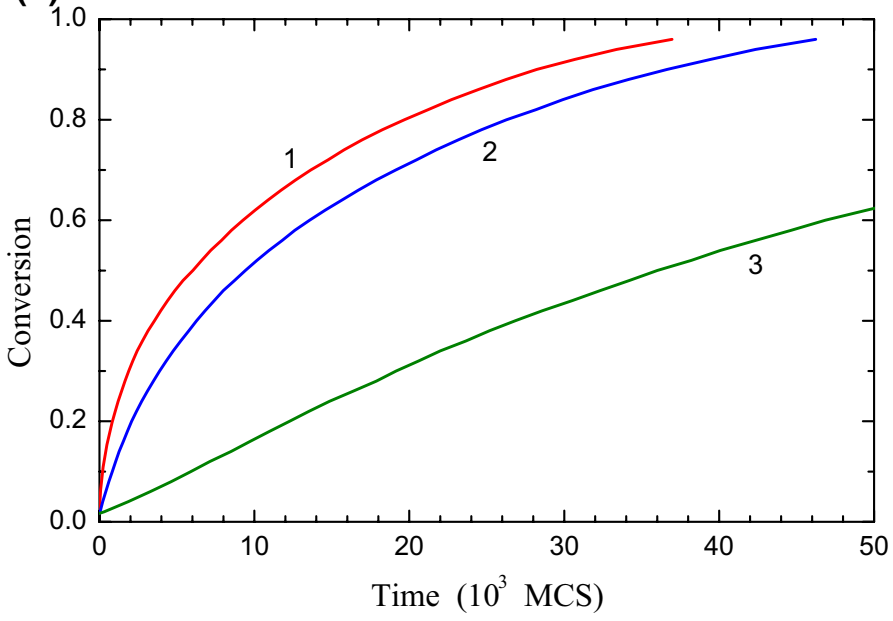

Fig. 1 Conversion to the oxide state as a function of time: $\mathbf{a}$ in the absence of grain boundaries with $p_{\mathrm{ox}}=1$ (curve 1; an example of the corresponding lattice snapshots is shown in Fig. 2) and in the presence of a grain boundary with rapid supply of IOIs via this boundary (so that the boundary sites at thermodynamic equilibrium with the gas-phase oxygen) and $p_{\mathrm{ox}}=1$ (curve 2 ; an example of the corresponding lattice snapshots is shown in Fig. 3); $\mathbf{b}$ in the presence of a grain boundary and the IOI jumps at the corresponding sites occuring with the same rate constant as on the regular oxide sites [curves 1,2 , and 3 are for $p_{\mathrm{ox}}=1$ (one of the corresponding lattice snapshots is shown in Fig. 4), 0.1 (the corresponding lattice snapshot is shown in Fig. 5), and 0.01 (the corresponding lattice snapshot is shown in Fig. 6), respectively] 


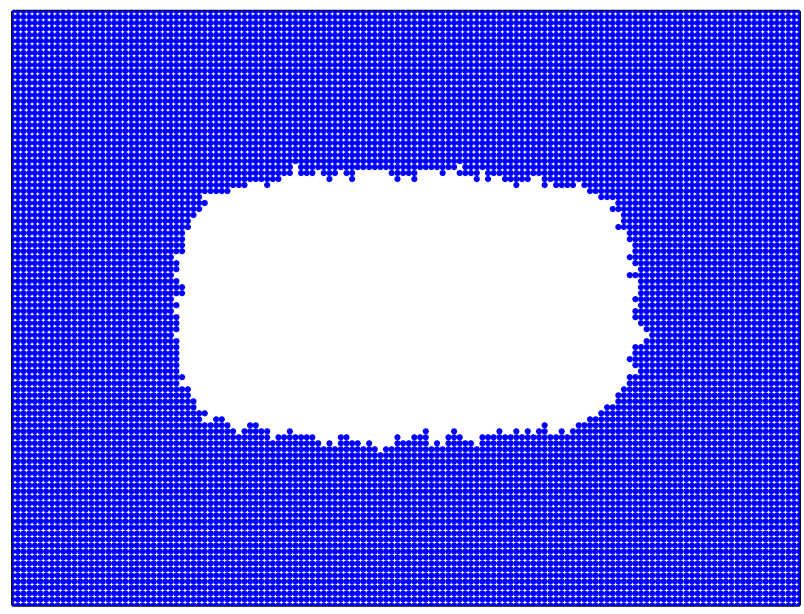

Fig. 2 Lattice snapshot corresponding to the kinetics in the absence of grain boundaries [curve 1 in Fig. 1a] at the moment when the conversion to the oxide state is equal to 0.75

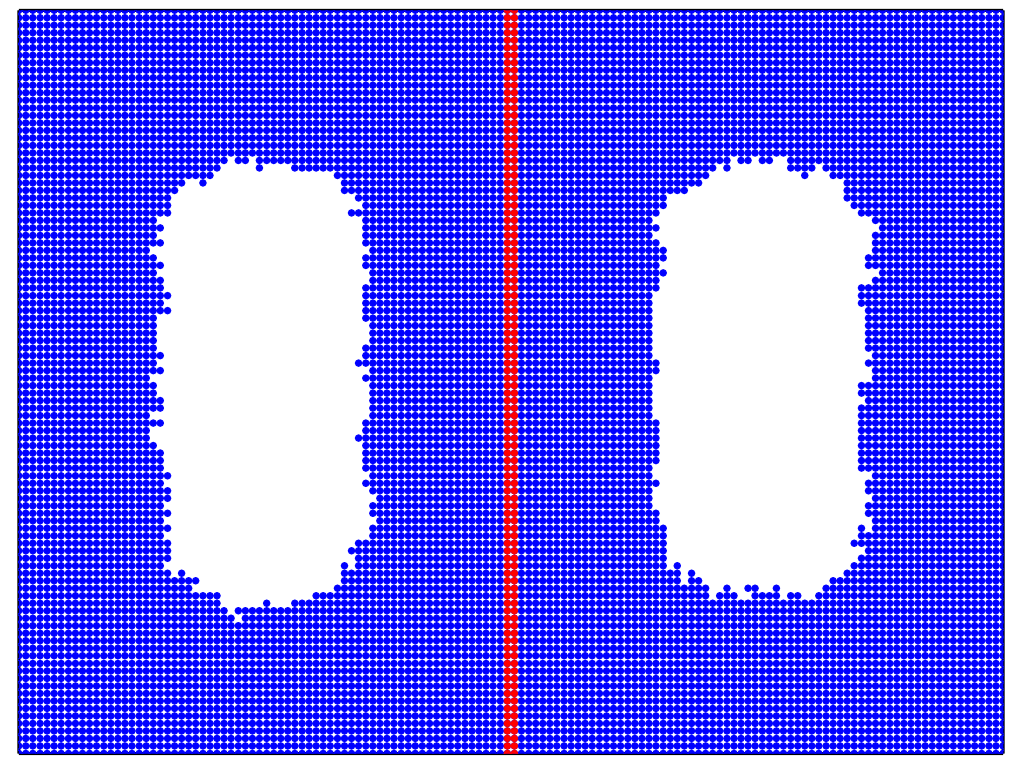

Fig. 3 As Fig. 2 for the kinetics in the presence of a grain boundary with rapid supply of IOIs via this boundary [curve 2 in Fig. 1b]

the conversion to the oxide state is equal to 0.75 . Additional lattice snapshots for the moments when the conversion to the oxide state is equal to 0.25 and 0.5 are shown in Supporting Information. 


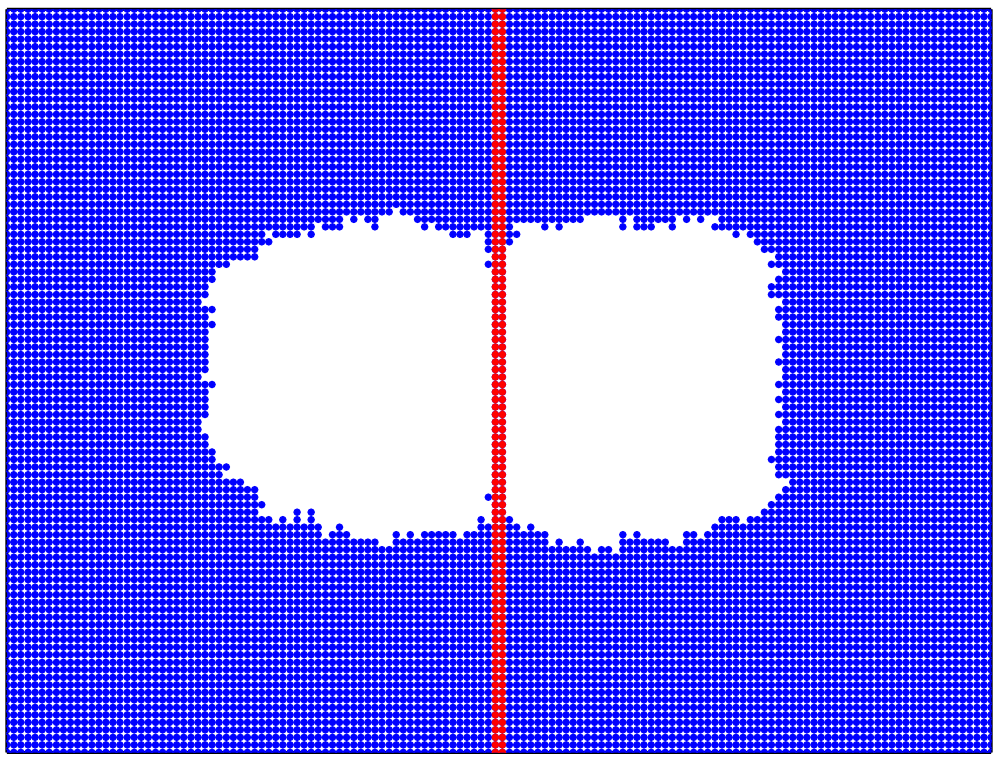

Fig. 4 As Fig. 3 with the IOA jumps occurring at the grain-boundary oxide sites with the same rate constant as on the regular oxide sites. The oxidation events are run with $p_{\mathrm{ox}}=1$ [curve $1 \mathrm{in} \mathrm{Fig.} 1 \mathrm{~b}$ ]

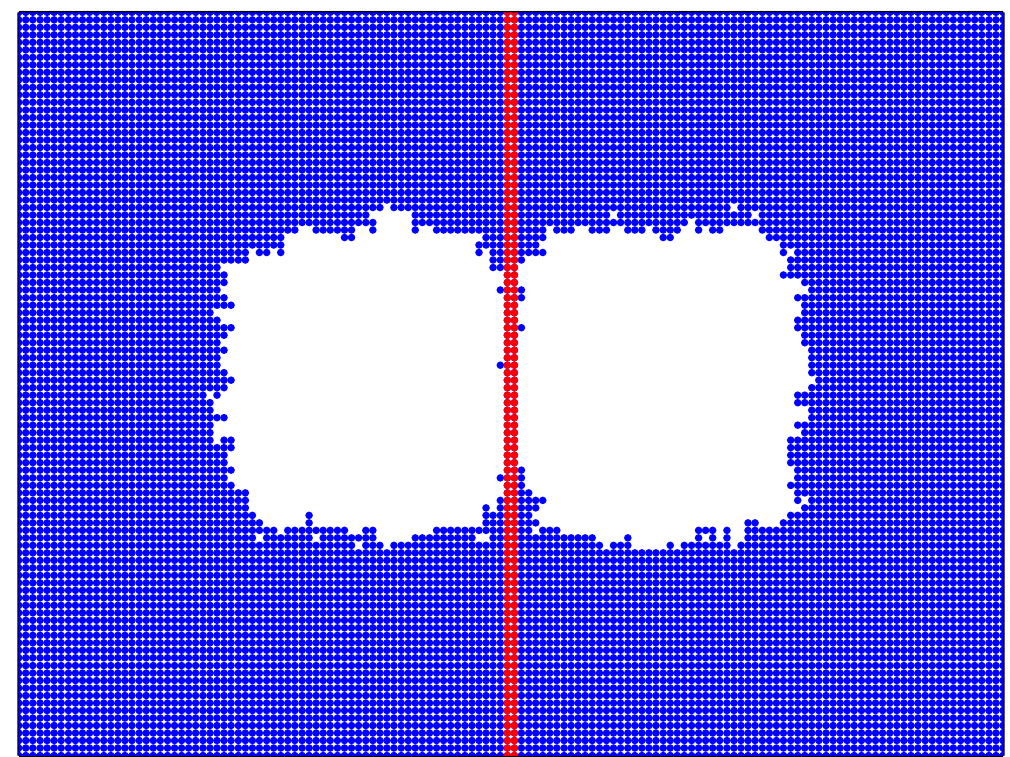

Fig. 5 As Fig. 4 for $p_{\text {ox }}=0.1$ [curve 2 in Fig. 1b]

In the absence of a grain boundary, the kinetics are close to parabolic [curve 1 in Fig. 1a with $p_{\mathrm{ox}}=1$ ], and the corresponding lattice snapshots (Fig. 2) show a shrinking metal core. 


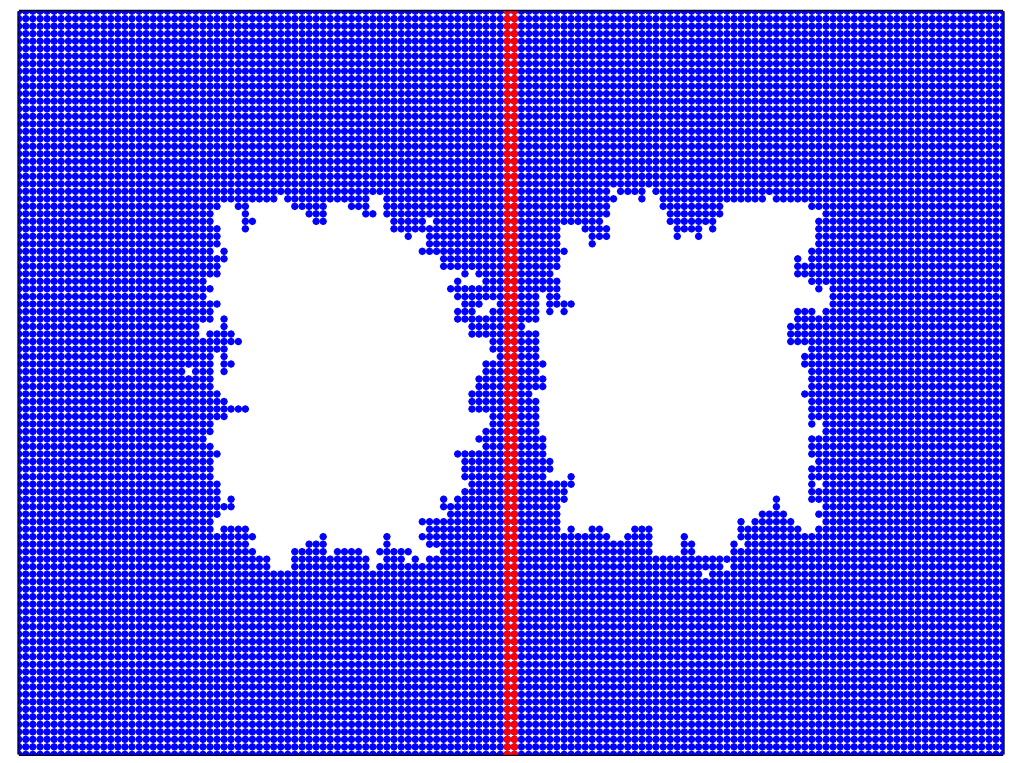

Fig. 6 As Fig. 4 for $p_{\text {ox }}=0.01$ [curve 3 in Fig. 1b]

In the presence of a grain boundary and with rapid supply of IOIs via this boundary (so that the boundary sites at thermodynamic equilibrium with the gas-phase oxygen) and $p_{\mathrm{ox}}=1$, the oxidation of two parts of the lattice occurs independently in the qualitatively similar regime as in the absence of a grain boundary [cf. curve 2 in Figs. 1a and 3 with curve 1 in Figs. 1a and 2].

If the IOI jumps at the grain-boundary oxide sites occur with the same rate constant as on the regular oxide sites, the kinetics and snapshots depend on the rate constant of oxidation, $p_{\mathrm{ox}}$. If the oxidation is rapid and this rate constant is equal to that of diffusion, i.e., $p_{\mathrm{ox}}=1$, the oxidation is fully controlled by the IOI diffusion in the oxide shell, the kinetics is parabolic [curve 1 in Fig. 1b], and the role of the grain boundary is nearly negligible (Fig. 4). With decreasing $p_{\text {ox }}$ down to 0.1 , the situation remains similar [curve 2 in Figs. $1 \mathrm{~b}$ and 5]. With further decrease of $p_{\mathrm{ox}}$ down to 0.01 , the kinetics is closer to liner [curve 3 in Fig. 1b] because the oxidation is kinetically controlled, the role of the grain boundary becomes appreciable, and the lattice snapshots (Fig. 6) are qualitatively similar to those observed in the case of independent oxidation of grains (Fig. 2).

\section{Conclusions}

The present MC simulations of oxidation of metal NPs are focused on the situation when the oxidation occurs via IOI diffusion in the oxide. The emphasis is on the role of the grain-boundary sites. During oxidation these sites are considered to be in the oxide state, and their role is demonstrated to be significant provided they are able to mediate rapid supply of IOIs (Fig. 2) or provided the oxidation 
is much slower (at least by two orders of magnitude) than IOI diffusion via the oxide (Fig. 6). The realization of these conditions is not simple. Oxidation is accompanied by the lattice expansion and reconfiguration, and physically there are no strong arguments in favour that in this case the grain-boundary oxide sites are highly efficient in the IOI supply. Energetically, the oxide formation is usually favourable, and the oxidation is not expected to be much slower than IOI diffusion.

Despite the reservations above, the already mentioned recent experiments performed by Maack and Nilius [16] are indicative of preferred oxidation along grain boundaries in polycrystalline $\mathrm{Cu}$ films. Thus, the regime of oxidation of NPs with rapid oxygen diffusion along grain boundaries and subsequent oxidation of each grain almost independently (as in Fig. 6) is expected to be likely at least in the case of $\mathrm{Cu}$ NPs. Oxidation of such NPs was experimentally studied at the ensemble level [30, 32] and also at the level of individual particles [33] by using plasmonic nanospectroscopy and electrodynamic simulations. The interpretation of the results was there based on the assumption that the oxidation occurs via the conventional mechanism including the formation of the oxide shell. The likely role of grains was not discussed. Concerning these and other experiments, I may add that at the ensemble level, the identification of the role of grains is hardly possible, because due to the difference in the grain structure, the shape and timescale of oxidation of different NPs are expected to be different, and accordingly the interpretation of the lumped kinetics corresponding to an array on NPs can be far from straightforward. Experiments with individual particles may be much more useful in this context.

Concerning the theory, I may notice that the lattice model used can easily be extended in various directions. The lattice simulations of oxidation of metal NPs occurring via diffusion of metal atoms in the oxide are also possible (some related results are available e.g. in Ref. [42]). The latter scenario is, however, more complex due to the likely formation of hollows (Kirkendall effect). The corresponding models should be more complex as well. In particular, the metal-metal interaction should be taken into account.

Another extension can be related to the interpretation of the oxidation kinetics measured at the ensemble level. From this perspective, it might be instructive to show theoretically how the size distributions of NPs in an array and grains in NPs are manifested in the lumped oxidation kitetics (for hydride formation in NPs, the role of the NP-size distribution was shown in Ref. [53]).

Finally, the model presented can be reformulated in terms corresponding to other processes. For example, the already mentioned hydride formation during absorption of hydrogen by metal NPs (e.g., by Pd) is usually considered to occur via the formation of a layer of a new phase near the gas-NP interface and its expansion inwards, and the whole process is often described by employing the conventional core-shell models [54-60]. Physically, this process is similar to oxidation, and accordingly the conclusions driven above for oxidation can be applicable to the hydride formation in metal NPs with grains. In this context, one can notice that the experimental studies of the specifics of hydride formation in metal NPs with grains are just beginning [61]. 
Acknowledgements Open access funding is provided by Chalmers University of Technology. I thank Christoph Langhammer for the collaboration (Ref. [32]) and useful discussions which induced this work.

Open Access This article is licensed under a Creative Commons Attribution 4.0 International License, which permits use, sharing, adaptation, distribution and reproduction in any medium or format, as long as you give appropriate credit to the original author(s) and the source, provide a link to the Creative Commons licence, and indicate if changes were made. The images or other third party material in this article are included in the article's Creative Commons licence, unless indicated otherwise in a credit line to the material. If material is not included in the article's Creative Commons licence and your intended use is not permitted by statutory regulation or exceeds the permitted use, you will need to obtain permission directly from the copyright holder. To view a copy of this licence, visit http://creativecommons.org/licen ses/by/4.0/.

\section{References}

1. Atkinson A (1985) Transport processes during the growth of oxide films at elevated temperature. Rev Mod Phys 57:437-470

2. Young DJ (2016) High temperature oxidation and corrosion of metals. Elsevier, Amsterdam

3. Tammann G (1920) Über Anlauffarben von Metallen. Zeitsch Anorg Allg Chem 111:78-89

4. Pilling NE, Bedworth RE (1923) The oxidation of metals at high temperatures. J Inst Metals 29:529-582

5. Wagner C (1933) Beitrag zur Theorie des Anlaufvorgangs. Z Phys Chem B 21:25-41

6. Valensi G (1936) Kinetics of oxidation of metallic spherules and powders. Comput Rend Acad Sci 202:309-312

7. Cabrera N, Mott NF (1949) Theory of the oxidation of metals. Rep Progr Phys 12:163-184

8. Carter RE (1961) Kinetic model for solid-state reactions. J Chem Phys 34:2010-2015

9. Fromhold AT (1964) Parabolic growth law for coherent oxides. J Chem Phys 41:509-514

10. Fehlner FP, Mott NF (1970) Low-temperature oxidation. Oxid Metals 2:59-99

11. Zhdanov VP, Norton PR (1996) Growth of oxide films on metal surfaces: transition from parabolic to linear kinetics. Appl Surf Sci 99:205-211

12. Kusano KF, Uchikoshi M, Mimura K, Isshiki M (2014) Low-temperature oxidation of Cu(100), $\mathrm{Cu}(110)$ and $\mathrm{Cu}(111)$. Oxid Metals 82:181-193

13. Cao F et al (2016) Atomistic observation of structural evolution during magnesium oxide growth. J Phys Chem C 120:26873-26878

14. Gorr B, Müller F, Azim M, Christ H-J, Müller T, Chen H, Kauffmann H (2017) High-temperature oxidation behavior of refractory high-entropy alloys: effect of alloy composition. Oxid Metals 88:339-349

15. Liu Q, Ning Y, Huang W, Fu Q, Yang F, Bao X (2018) Origin of the thickness-dependent oxidation of ultrathin Cu films on Au(111). J Phys Chem C 122:8364-8372

16. Maack B, Nilius N (2019) In-situ optical view onto copper oxidation-role of reactive interfaces and self-heating. Corros Sci 159:108112

17. Auinger M, Naraparaju R, Christ H-J, Rohwerder M (2011) Modelling high temperature oxidation in iron-chromium systems: combined kinetic and thermodynamic calculation of the long-term behaviour and experimental verification. Oxid Metals 76:247-258

18. Garcia Martin JF, Sanchez S, Metz R (2012) Kinetics model of the thermal oxidation of indium powder. Oxid Metals 77:1-7

19. Leblond J-B, Pignol M, Huin D (2013) Predicting the transition from internal to external oxidation of alloys using an extended Wagner model. C R Mecanique 341:314-322

20. Zaeem MA, El Kadiri H (2014) An elastic phase field model for thermal oxidation of metals: application to zirconia. Comput Mater Sci 89:122-129

21. Wang H, Suo Y, Shen S (2015) Reaction-diffusion-stress coupling effect in inelastic oxide scale during oxidation. Oxid Metals 83:507-519

22. Fang X, Dong X, Jiang D, Feng X (2018) Modification of the mechanism for stress-aided grain boundary oxidation ahead of cracks. Oxid Metals 89:331-338 
23. Lin C, Ruan H (2019) Phase-field modeling of scale roughening induced by outward growing oxide. Materialia 5:100255

24. Batailloua L, Desgrangesa C, Martinellia L, Monceauc D (2018) Modelling of the effect of grain boundary diffusion on the oxidation of Ni-Cr alloys at high temperature. Corros Sci 136:148-160

25. Geers C, Panas I (2019) Impact of grain boundary density on oxide scaling revisited. Oxid Metals 91:55-75

26. Yang Z, Yang N, Pileni M-P (2015) Nano Kirkendall effect related to nanocrystallinity of metal nanocrystals: influence of the outward and inward atomic diffusion on the final nanoparticle structure. J Phys Chem C 119:22249-22260

27. Förster GD et al (2015) Oxidation-induced surface roughening of aluminum nanoparticles formed in an ablation plume. Phys Rev Lett 115:246101

28. Litrico G, Proulx P, Gouriet J-B, Rambaud P (2015) Controlled oxidation of aluminum nanoparticles. Adv Powder Technol 26:1-7

29. Han L et al (2016) Interrogation of bimetallic particle oxidation in three dimensions at the nanoscale. Nat Commun 7:13335

30. Susman MD, Vaskevich A, Rubinstein I (2016) A general kinetic-optical model for solid-state reactions involving the nano Kirkendall effect. The case of copper nanoparticle oxidation. J Phys Chem C 120:16140-16152

31. Yu J, Yuan W, Yang H, Xu Q, Wang Y, Zhang Z (2018) Fast gas-solid reaction kinetics of nanoparticles unveiled by millisecond in situ electron diffraction at ambient pressure. Angew Chem Intern Ed 57:11344-11348

32. Albinsson D, Nilsson S, Antosiewicz TJ, Zhdanov VP, Langhammer C (2019) Heterodimers for in situ plasmonic spectroscopy: $\mathrm{Cu}$ nanoparticle oxidation kinetics, Kirkendall effect, and compensation in the Arrhenius parameters. J Phys Chem C 123:6284-6293

33. Nilsson S, Albinsson D, Antosiewicz TJ, Fritzsche J, Langhammer C (2019) Resolving single Cu nanoparticle oxidation and Kirkendall void formation with: In situ plasmonic nanospectroscopy and electrodynamic simulations. Nanoscale 11:20725-20733

34. Gao J, Yan J, Zhao B, Zhang Z, Yu Q (2020) (2020) In situ observation of temperature-dependent atomistic and mesoscale oxidation mechanisms of aluminum nanoparticles. Nano Res 13:183-187

35. Svoboda J, Fischer FD, Vollath D (2009) Modeling of formation of binary-phase hollow nanospheres from metallic solid nanospheres. Acta Mater 57:1912-1919

36. Zhdanov VP, Kasemo B (2009) On the feasibility of strain-induced formation of hollows during hydriding or oxidation of metal nanoparticles. Nano Lett 9:2172-2176

37. Svoboda J, Fischer FD (2011) Stress development during reaction of metallic nanospheres with gas. Acta Mater 59:61-67

38. Zhdanov VP, Kasemo B (2008) Cabrera-Mott kinetics of oxidation of nm-sized metal particles. Chem Phys Lett 452:285-288

39. Ermoline A, Dreizin EL (2011) Equations for the Cabrera-Mott kinetics of oxidation for spherical nanoparticles. Chem Phys Lett 505:47-50

40. Zhdanov VP, Kasemo B (2012) Cabrera-Mott kinetics of oxidation of metal nanowires. Appl Phys Lett 100:243105

41. Zhdanov VP (2017) Oxidation of metal nanoparticles with the grain growth in the oxide. Chem Phys Lett 674:136-140

42. Zhdanov VP (2019) Kirkendall effect in the two-dimensional lattice-gas model. Phys Rev E 99:012132

43. Zhdanov VP (2019) Kinetic model of oxidation of metal nanoparticles: Cabrera-Mott and Kirkendall effects. Surf Sci 684:24-27

44. Yin Y, Rioux RM, Erdonmez CK, Hughes S, Somorjal GA, Alivisatos AP (2004) Formation of hollow nanocrystals through the nanoscale Kirkendall effect. Science 304:711-714

45. Fan HJ, Gösele U, Zacharias M (2007) Formation of nanotubes and hollow nanoparticles based on Kirkendall and diffusion processes: a review. Small 3:1660-1671

46. Mehrer H (2007) Diffusion in solids: fundamentals, methods, materials, diffusion-controlled processes. Springer, Berlin

47. Lacaille V, Morel C, Feulvarch E, Kermouche G, Bergheau J-M (2014) Finite element analysis of the grain size effect on diffusion in polycrystalline materials. Comput Mater Sci 95:187-191

48. Moghadam MM, Rickman JM, Harmer MP, Chan HM (2015) The role of boundary variability in polycrystalline grain-boundary diffusion. J Appl Phys 117:045311 
49. Payne BP, Grosvenor AP, Biesinger MC, Kobe BA, McIntyre NS (2007) Structure and growth of oxides on polycrystalline nickel surfaces. Surf Interfaces Anal 39:582-592

50. Lisoni JG, Goux L, Hoffmann Th, Diaz-Droguett DE, Jurczak M (2012) Influence of the microstructure on the oxidation of Ni thin films. Corros Sci 59:282-289

51. Chen Y, An X, Liao X (2017) Mechanical behaviors of nanowires. Appl Phys Rev 4:031104

52. Zhdanov VP (2019) Relaxation of a dislocation in a nanocrystallite. Phys Lett A 383:744-747

53. Zhdanov VP, Kasemo B (2009) Apparent kinetics of hydriding and dehydriding of metal nanoparticles. Physica E 42:1482-1486

54. Zhdanov VP, Kasemo B (2010) Kinetics of the formation of a new phase in nanoparticles. Chem Phys Lett 460:158-161

55. Zhdanov VP, Kasemo B (2009) The formation of a new phase in nanoparticles. Physica E 41:775-778

56. Zhdanov VP (2010) Effect of lattice strain on the kinetics of hydride formation in metal nanoparticles. Chem Phys Lett 492:77-81

57. Langhammer C, Zhdanov VP, Zorić I, Kasemo B (2010) Size-dependent kinetics of hydriding and dehydriding of Pd nanoparticles. Phys Rev Lett 104:135502

58. Bardhan R, Hedges LO, Pint CL, Javey A, Whitelam S, Urban JJ (2013) Uncovering the intrinsic size dependence of hydriding phase transformations in nanocrystals. Nat Mater 12:905-912

59. Sun X, Ariza P, Ortiz M, Wang KG (2018) Long-term atomistic simulation of hydrogen absorption in palladium nanocubes using a diffusive molecular dynamics method. Int $\mathbf{J}$ Hydr Energy 3:5657-5667

60. Sun X, Ariza MP, Ortiz M, Wang KG (2019) Atomistic modeling and analysis of hydride phase transformation in palladium nanoparticles. J Mech Phys Sol 125:360-383

61. Alekseeva $\mathrm{S}$ et al (2017) Grain boundary mediated hydriding phase transformations in individual polycrystalline metal nanoparticles. Nat Commun 8:1084

Publisher's Note Springer Nature remains neutral with regard to jurisdictional claims in published maps and institutional affiliations. 